\title{
A Platform to Improve Echinococcosis Control in Tibetan Populations - Sichuan Province, China, 2015-2020
}

\author{
Tian Tian'; Shuai Han'; Chuizhao Xue'; Xu Wang'; Shijie Yang'; Bo Zhong²; Qian Wang²; Wenjie Yu²;

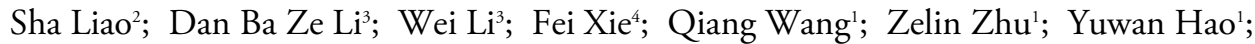 \\ Weiping $\mathrm{Wu}^{1}$; Ning Xiao'; Xiao-nong $\mathrm{Zhou}^{1, *}$
}

Echinococcosis is a neglected parasitic zoonotic disease that is distributed worldwide. Previous data has shown that China holds the highest disease burden of echinococcosis in the world, resulting in $22.97 \times 10^{5}$ disability adjusted life year (DALYs) lost annually (1). Echinococcosis is one of the epidemiological factors responsible for poverty in China. Since 2000, China's central government (CG) has taken several actions to control echinococcosis in Western China. Since 2005, special funds by the CG have been allocated to echinococcosis research. Since 2007, echinococcosis has been listed as 1 of the 6 communicable diseases that exempts medical expenses. In 2010, the Action Plan for the Prevention and Control of Echinococcosis (2010-2015) [APPCE (2010-2015)] was issued by the CG (2-3).

Ganzi Tibetan Autonomous Prefecture is located in western Sichuan Province with an average altitude of over 4,000 meters above sea level, and $97 \%$ of the population is Tibetan. Ganzi is an area with one of the highest disease burdens of endemic echinococcosis, and echinococcosis is prevalent in all 18 counties across Ganzi. In November 2015, China CDC established a work office to support the echinococcosis control program to reduce the associated morbidity and mortality. During 2016-2020, a series of control interventions has been implemented to control the echinococcosis transmission under the leadership of the work office: 1) an integrated control strategy has been implemented in 3 townships covering $1.53 \times 10^{5}$ square kilometers; 2) the morbidity rate has been reduced by $86.18 \%$; 3) a total of 1,000 local professionals and technical staff have been trained for echinococcosis control; and 4) cultivation of health literacy has covered a population of 1.1 million. So far, echinococcosis remains highly prevalent in western China; the Ganzi work office might provide valuable experience to support the elimination of echinococcosis.

\section{PROMOTION OF THE ECHINOCOCCOSIS CONTROL PROGRAM IN GANZI}

As one of the most severely affected areas, endemic echinococcosis is still transmitted in all 18 counties across Ganzi. According to the 2012 National Echinococcosis Survey, there was a echinococcosis prevalence rate of $12.09 \%$ in humans in Shiqu County, which was far greater than the average level in Sichuan Province $(1.08 \%)$ and nationwide $(0.24 \%)$. To effectively eliminate the effects on health and alleviate the poverty resulting from echinococcosis in Ganzi, China's former Ministry of Health [currently named the National Health Commission (NHC)] and Sichuan Provincial Government launched a joint project for controlling echinococcosis in 2010. The NHC issued the Comprehensive Prevention and Control Pilot Program for Echinococcosis in Shiqu County, Ganzi Prefecture, Sichuan Province (2016-2020) [CPCPPESC, GPSP (2016-2020)] that aims to effectively control echinococcosis transmission in Shiqu County through the implementation of integrated control measures (4-7).

The primary purposes of the work office established by China CDC includes the following: (1) understanding the current prevalence of echinococcosis in Ganzi Prefecture through epidemiological investigations; (2) facilitating echinococcosis control by means of a systematic package of interventions including the dog deworming, health education, and mass drug administration of patients, etc.; (3) researching and developing novel vaccines and drugs for echinococcosis; and (4) improving the capacity building of local grass-roots professionals.

During 2016-2020, a total of 25.717 million CNY (roughly 3.97 million USD) were allocated to the pilot program by the NHC and China CDC, and 49 subprojects were performed during this period 
supervised by the National Institute of Parasitic Diseases (NIPD) of China CDC (5). In terms of the number of subprojects implemented, the subprojects covered the fields of control strategy (19.6\%), fundamental research $(10.7 \%)$, drug research and development (drug R\&D) (14.3\%), detection reagent development $(5.4 \%)$, diagnostic reagent development $(21.4 \%)$, vaccine research $(8.9 \%)$, biobank construction (14.3\%), and health education $(5.4 \%)$ (Figure 1).

\section{ROLES OF THE WORK OFFICE IN GANZI}

In the pilot subproject of control strategy carried out in 3 townships of Shiqu County of Ganzi, epidemiological data indicated that the implementation of the pilot subprojects increased the awareness rate of echinococcosis control in Shiqu County from $41.2 \%$ (247/600 participants) in 2016 to $91.3 \%(210 / 230)$ in 2019 , and greatly reduced the number of affected domestic dogs from 52,229 in 2016 to 15,981 in 2019 . The positive rates of echinococcosis in dog excrement were $1.24 \%$ (8/644 samples) in 2016 and $1.67 \%$ (1/60) in 2019.

For drug R\&D, albendazole and mebendazole soft capsules have been developed. New promising anti- echinococcosis formulations improved the bioavailability of the drugs and reduced the required dosages, which reduced the side effects of the drugs. In the aspect of detection and diagnostic reagents, three recombinant Echinococcus antigens were applied for combined diagnosis. The technology included a colloidal gold rapid detection kit for echinococcosis and a mobile traceable system with high diagnostic efficiency, simple production, low costs, ease of use, real-time analysis, and feedback and storage of test results; however, this set up could not distinguish cystic echinococcosis (CE) from alveolar echinococcosis (AE).

For biobank construction, more than 1,000 samples (800 serum samples, 200 other biological samples: cysts, tissues, feces, urines) have been collected and standardized for storage. In addition, health education was carried out in 6 schools, and nearly 1,000 people were investigated for their awareness levels after the intervention. Health education was strengthened, and more than 1,200 echinococcosis educational supplies were distributed.

In August 2020, the work office, along with the 45 subprojects, was evaluated by a panel of experts from hospitals, disease control institutions, and health administrations. Results from the final evaluation report revealed the success of the work office by having an evaluation score of 94.13 out of a total of 100 score

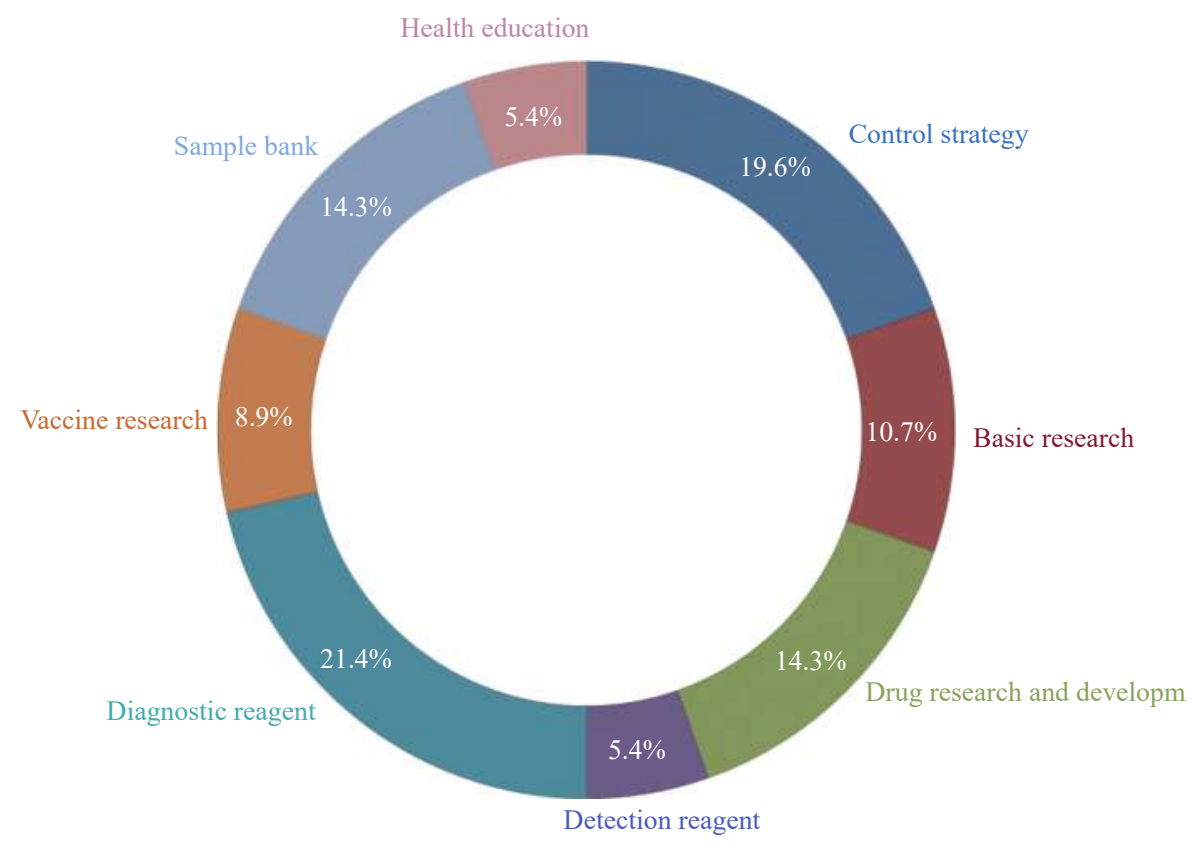

FIGURE 1. Research topics of the subprojects for the comprehensive echinococcosis prevention and control in Ganzi from 2016 to 2020 *.

* The percentage presented in the figure is the proportion of the total number of investigation subprojects. 
and demonstrating the major proposed objectives have been achieved.

\section{DISCUSSION}

The promotion of echinococcosis control in the Tibet areas has been launched through the implementation of the CPCPPESC, GPSP (2016-2020). Under this pilot program, financial support, technical assistance, infrastructure construction, and capacity building were targeted for echinococcosis control in local areas in Ganzi. A total of 103 echinococcosis rehabilitation and treatment centers, livestock slaughtering centers, echinococcosis prevention and control laboratories, and safe water centers have been built in Shiqu County during the past 5 years, incurring a total cost of more than 1 billion CNY (8-11).

Based on the "pilot first" and "point-to-area" principle, the China CDC work office has played a critical role in implementing echinococcosis field control activities since initiation. Significant achievements in echinococcosis control realized in Ganzi were characterized by great reductions in the prevalence of echinococcosis in humans and domestic and wildlife hosts, remarkable improvements in awareness and behaviors pertaining to echinococcosis control, and significantly improved capacity building (Table 1).

However, there were still some challenges for the elimination of this disease in local areas. First, financial support should be enhanced. Currently, operational funds for the China CDC work office mainly depend on the CPCPPESC, GPSP (2016-2020), and limited financial support was obtained from other sources. A large number of transportation fees were reported (accounting for $27.74 \%(1,387,100 / 5,000,000 \mathrm{CNY})$ of the budget) during the implementation of field control activities due to the special climate in highaltitude areas. In addition, the current workspace of the China CDC work office primarily relies on the Ganzi CDC. The harsh high-altitude natural environments require higher levels of protection during echinococcosis field control activities. All these programs require more financial support.

Second, the integrated control strategy should be intensified. Following the implementation of the 5year pilot project of control, great achievements have been reached in echinococcosis control such as the reduction of morbidity to 0 in children aged 6-12 years among new patients. However, a risk factor of dog reinfection still exists, and it is difficult to construct shelters for stray dogs. Meanwhile, with the implementation of the National Ecological Protection Policy, increasing wildlife density could be a potential risk factor leading to echinococcosis transmission from wildlife to local human communities. Therefore, technical support is needed from stakeholders in animal husbandry, public security, and forestry administration. Consequently, a multidisciplinary collaboration mechanism needs to be built, and the integrated control strategy for echinococcosis should be reinforced (12-13).

Third, the translation of scientific research for echinococcosis into the clinic or field should be increased. During the operation of the 5-year pilot

TABLE 1. Evaluation of pilot targets and indicators for comprehensive echinococcosis control in Shiqu County in 2020.

\begin{tabular}{|c|c|c|c|c|}
\hline Category & $\begin{array}{c}\text { Baseline data in } \\
2014\end{array}$ & End of 2020 & $\begin{array}{c}\text { Results of self- } \\
\text { assessment in } 2020\end{array}$ & $\begin{array}{l}\text { On-site technical } \\
\text { evaluation results }\end{array}$ \\
\hline New patient detection rate in children aged 6-12 & $2.91 \%$ & Below $1 \%$ & $0 \%$ & $0 \%$ \\
\hline Canine infection rate & $6 \%$ & Below 2\% & $0.86 \%$ & $0.45 \%, 0.86 \%{ }^{*}$ \\
\hline Livestock infection rate & $45 \%$ & Below 25\% & $8.84 \%$ & $8.84 \%$ \\
\hline Domestic dog registration rate & $72 \%$ & Above $85 \%$ & $100 \%$ & $100 \%, 100 \%{ }^{*}$ \\
\hline Canine deworming coverage & $73 \%$ & $90 \%$ & $100 \%$ & $100 \%, 100 \%{ }^{*}$ \\
\hline Treatment rate of diseased organs in slaughterhouse & $-{ }^{\dagger}$ & $100 \%$ & $100 \%$ & $100 \%$ \\
\hline Safe drinking water coverage in settlements & $80 \%$ & $100 \%$ & $100 \%$ & $100 \%, 100 \% *$ \\
\hline Rodent control rate in settlements and within $1 \mathrm{~km}$ radius & $-\mathbf{-}^{\dagger}$ & $80 \%$ & $80 \%$ & $100 \%$ \\
\hline Awareness rate of prevention and control knowledge & $30 \%$ & Above $85 \%$ & $98.13 \%$ & $98.13 \%$ \\
\hline Standardized treatment rate of patients & $50 \%$ & Above $85 \%$ & $93.15 \%$ & $93.15 \%, 100 \%{ }^{*}$ \\
\hline Qualification rate of professional skills & $60 \%$ & $95 \%$ & $99.85 \%$ & $99.85 \%, 100 \%{ }^{*}$ \\
\hline
\end{tabular}

\footnotetext{
*Assessment of village level.
}

$\dagger$ No data. 
program, 20 national patents pertaining to echinococcosis were obtained. However, these patents are public welfare products, which lack the market collaboration mechanism to translate the patent into products. Currently, the work office is focused on epidemiological surveys of echinococcosis, assessing the risk of transmission of echinococcosis, and assessing the disease burden of echinococcosis; more attention should be given to the translation of the scientific research in the future. In addition, the work office requires the establishment of stronger collaborations with China CDC, most notably the field epidemiology training program, laboratory management, and continuing education management.

To respond to these challenges, the following suggestions are recommended. First, internal evaluations have proved the feasibility of the current operation model of the China CDC work office; therefore, we need to upgrade the current model, build a long-term mechanism, and continue to maintain a close collaboration with the expert team. Second, since echinococcosis is defined as a contributor to poverty, elimination of echinococcosis is critical to poverty alleviation. Therefore, poverty alleviation and echinococcosis control may be jointly performed to help the elimination program, build health services, and promote socioeconomic development. Third, the scientific research of echinococcosis needs to be translated into disease control products and services that are used to solve problems in the field. In addition, we need to make full use of national, intraprovincial, and extra-provincial experts in the prevention and control of echinococcosis to build a base of scientific innovation in the work office. Applied and operational studies are strongly recommended to attract market resources and funds for echinococcosis control programs. Finally, novel approaches to solve the problems of inconveniences in transportation and storage and laboratory testing of animal host samples are urgently required. Further epidemiological studies are required to investigate the transmission dynamics of wild animal echinococcosis in natural foci, to identify its risk factors, and to improve the integrated echinococcosis control in these target areas.

Acknowledgements: Participants of the national work office; National Health Commission of China; and China CDC.

\section{doi: $10.46234 / \mathrm{ccdcw} 2021.024$ \\ \# Corresponding author: Xiao-nong Zhou, zhouxn1@chinacdc.cn.}

\footnotetext{
${ }^{1}$ National Institute of Parasitic Diseases, Chinese Center for Disease
}

Control and Prevention; Chinese Center for Tropical Diseases Research; WHO Collaborating Centre for Tropical Diseases; National Center for International Research on Tropical Diseases, Ministry of Science and Technology; Key Laboratory of Parasite and Vector Biology, Ministry of Health, Shanghai, China; ${ }^{2}$ Sichuan Center for Disease Control and Prevention, Chengdu, Sichuan, China.; ${ }^{3}$ Center for Disease Control and prevention of Ganzi Tibetan Autonomous Prefecture, Ganzi, Sichuan, China.; ${ }^{4}$ Shiqu County Center for Disease Control and Prevention, Ganzi, Sichuan, China..

Submitted: October 09, 2020; Accepted: January 27, 2021

\section{REFERENCES}

1. Zhang MY, Wu WP, Guan YY, Wang LY, Hou YY, Feng Y, et al. Analysis on disease burden of hydatid disease in China. Chin J Parasitol Parasit Dis 2018;36(1): 15 - 9, 25. http://www.jsczz.cn/CN/ Y2018/V36/I1/15. (In Chinese).

2. WHO. Echinococcosis. Geneva: World Health Organization. 2020. http://www.who.int/zh/news-room/fact-sheets/detail/echinococcosis. [2020-3-23].

3. Tian T. Investigation on disease burden of echinococcosis in high endemicity areas of Qinghai-Tibet Plateau[dissertation]. Beijing: Chinese Center for Disease Control and Prevention; 2018. http://cdmd. cnki.com.cn/Article/CDMD-84501-1018309034.htm. (In Chinese).

4. Wu WP, Wang H, Wang Q, Zhou XN, Wang LY, Zheng CJ, et al. A nationwide sampling survey on echinococcosis in China during 2012-2016. Chin J Parasitol Parasit Dis 2018;36(1): 1 - 14. http://www. jsczz.cn/CN/Y2018/V36/I1/1. (In Chinese).

5. Wang GQ. Epidemiological survey on echinococcosis in China. Shanghai: Shanghai Scientific and Technical Publishers. 2016. http:// www.sjfx.com/books/view.aspx?id=2020100001007344. (In Chinese).

6. Tian T, Cao CL, Wu WP, Jia TW, Qian MB, Hao YW, et al. Epidemiological characteristics of echinococcosis death cases in China from 2008 to 2016. Chin J Schisto Control 2018;30(3): 282 - 8 . http://www.zgxfzz.com/CN/abstract/abstract10763.shtml. (In Chinese).

7. Fu MH, Han S, Xue CZ, Wang X, Liu BX, Wang Y, et al. Contribution to the echinococcosis control programme in China by NIPD-CTDR. Adv Parasitol 2020;110:107 - 44. http://dx.doi.org/ 10.1016/bs.apar.2020.04.010.

8. Sichuan Development and Reform Commission. The construction funds of comprehensive prevention and control project of hydatidosis were all in place. 2017. http://fgw.sc.gov.cn//sfgw/xmgla/2017/5/18/5b 918cd40504470997701a4c58f44c62.shtml. [2020-11-1]. (In Chinese).

9. Sichuan Provincial Government. Sichuan invested 176 million yuan to control hydatidosis this year, and the screening rate of target population reached 6.64\%2018http://www.sc.gov.cn/10462/10464/10797/2018/9/ 30/10460060.shtml. [2020-11-1]. (In Chinese).

10. Health News. Shiqu: herdsmen no longer talk about "hydatid" fear. 2017. http://www.jkb.com.cn/news/depth/2017/0617/412528.html. [2020-11-1]. (In Chinese).

11. Sichuan Provincial Government. Build one belt, one road, the 13 countries of the prevention and control network of hydatid disease, and sign the Chengdu declaration. 2017. http://www.sc.gov.cn/10462/10464/ 10797/2017/11/16/10438352.shtml. [2020-11-1]. (In Chinese).

12. Tian KQ, Tian T, Shi GQ, Cao CL, Hao YW, Zhu ZL, et al. Investigation on unhealthy related behaviors of echinococcosis patients in Garzê Prefecture, Sichuan Province. Chin J Schisto Control 2018;30(2): 165 - 72, 183. http://www.zgxfzz.com/CN/Y2018/V30/ I2/165. (In Chinese).

13. Xu GR, Zhang LJ, Zeng G. Epidemic analysis of echinococcosis in Ganzi Tibetan autonomous prefecture of Sichuan Province from 2006 to 2011. Chin J Parasitol Parasit Dis 2013;31(3): 224-8. http://www. jsczz.cn/CN/Y2013/V31/I3/14. 